\title{
Simulação do comportamento dinâmico de um conjunto trator-colhedora de feijão ${ }^{1}$
}

\author{
Ricardo F. Garcia ${ }^{2}$, Daniel M. de Queiroz ${ }^{3}$, Haroldo C. Fernandes ${ }^{4}$ \& Olímpio H. Miyagaki ${ }^{5}$ \\ 1 Parte da Tese de Doutorado do promeiro autor apresentada no Prog. de Pós-Graduação em Eng. Agrícola, UFV \\ 2 UENF. CEP 28013-600, Campo dos Goytacases, RJ. Fone: (22) 2726-1543. E-mail: garcia@uenf.br (Foto) \\ 3 DEA/UFV. CEP 36571-000, Viçosa, MG. Fone: (31) 3899-1882. E-mail: queiroz@ufv.br \\ ${ }^{4}$ DEA/UFV. Fone: (31) 3899-1883. E-mail: haroldo@ufv.br \\ ${ }^{5}$ Depto. de Matemática/UFV. Fone: (31) 3899-3011. E-mail: olimpio@ufv.br
}

Protocolo 171 - 13/11/2002 - Aprovado em 1/9/2003

\begin{abstract}
Resumo: No Brasil, a colheita mecanizada do feijão é realizada em duas etapas, em que na primeira, o produto é arrancado e enleirado manualmente e, na segunda etapa, ele é colhido, trilhado e limpo, usando-se uma colhedora de feijão, geralmente puxada por um trator. $\mathrm{O}$ aprimoramento do projeto dessas máquinas tem sido realizado por tentativa e erro. Neste trabalho, apresenta-se um modelo de simulação para analisar o comportamento dinâmico de um conjunto trator-colhedora de feijão, desenvolvido usando-se o programa de computador ADAMS, versão 10.0. Este modelo incluiu a simulação do trator, interação entre solo e pneu, simulação do sistema de transmissão de potência e dos sistemas de trilha e limpeza da colhedora de feijão. Finalmente, foram realizadas análises do comportamento dinâmico do conjunto trator-colhedora de feijão, sob duas diferentes condições de simulação, a fim de validar o modelo desenvolvido. Os resultados obtidos na simulação foram comparados com os obtidos em testes experimentais realizados em condições de campo. Na melhor condição de simulação, observou-se erro relativo médio de $8,18 \%, 5,85 \%, 28,69 \%$ e 26,23\%, para a velocidade de deslocamento, rotação da TDP, torque requerido na TDP e potência requerida na TDP, respectivamente. Em outra condição de simulação, o modelo simulou melhor a taxa de alimentação total, observando-se erro relativo médio de 4,99\%.
\end{abstract}

Palavras-chave: ADAMS, colheita, modelagem

\section{Simulation of dynamic performance of a tractor-harvester system for beans}

\begin{abstract}
Bean crop is generally harvested in two stages in Brazil. In the first one the product is manually pulled out and in the second stage the product is picked-up, threshed and cleaned using a bean harvester. The harvesters are generally tractor pulled. The design optimization of these machines has been done by trial and error. In this paper, a simulation model is presented to analyze the dynamic performance of a tractor-harvester system for beans. A simulation model was developed using ADAMS version 10.0. The model includes tractor simulation and a soil/ tyre interface, a power train and threshing and cleaning systems of the bean harvester. Finally, the dynamic performance of the tractor-harvester system for beans was evaluated using two modeling conditions. Comparing actual and simulated data, under the best conditions, the mean relative errors of $8.18,5.85,28.69$ and $26.23 \%$, respectively, for the forward speed, PTO rotational speed, PTO required torque and PTO required power were found. In another simulation condition, the mean relative error of $4.99 \%$ for the total feeding rate was observed.
\end{abstract}

Key words: ADAMS, harvest, modeling

\section{INTRODUÇÃO}

O Brasil é considerado, grande produtor de feijão (Phaseolus vulgaris L.) tanto em produção como em área cultivada atingindo, na safra 2001/2002, segundo previsões da CONAB (Companhia Nacional de Abastecimento), 3,26 milhões de toneladas, com área plantada de 4,21 milhões de hectares (Brasil, 2002).
Durante seu cultivo e segundo Conto et al. (1980) a quase totalidade dos agricultores utiliza a prática de colheita manual, que consiste em arrancar as plantas inteiras quando elas se encontram quase despidas de folhas. As plantas arrancadas permanecem na lavoura para completar o processo de secagem e, em seguida, são transportadas para um terreiro, onde é feita a trilha, utilizando-se bateção manual ou mecânica 
ou, então, são enleiradas na própria lavoura, para recolhimento mecânico.

A colhedora de feijão que recolhe, trilha, separa e limpa os grãos de feijão, é acionada e puxada por um trator. Existem algumas indústrias produzindo este tipo de máquina, no Brasil. Essas máquinas são desenvolvidas por tentativa e erro, mas seu projeto pode ser aperfeiçoado utilizando-se alguma técnica de projeto auxiliado por computador, como a simulação de sistemas mecânicos. Com esta técnica, a máquina é dividida em vários corpos rígidos que são interligados por juntas apropriadas, sendo as equações de movimento obtidas e solucionadas numericamente.

O programa comercial utilizado na simulação de sistemas mecânicos, geralmente divide o problema nas fases de préprocessamento, análise e pós-processamento (Queiroz, 1995). $\mathrm{Na}$ fase de pré-processamento, o usuário define os corpos rígidos e suas propriedades, as forças externas aplicadas e a forma pela qual os corpos estão ligados e na fase de solução o usuário define o método e seus parâmetros e executa a análise. O programa gera, automaticamente, as equações de movimento e as soluciona. $\mathrm{Na}$ fase de pós-processamento, o usuário pode ver os resultados através da animação do movimento, da plotagem de dados ou da visualização de valores numéricos da simulação.

Desta forma, este trabalho foi desenvolvido com o objetivo de usar a técnica de simulação de sistemas mecânicos para desenvolver um modelo de simulação de um conjunto tratorcolhedora de feijão, utilizando o programa de computador ADAMS; analisar o comportamento dinâmico do conjunto trator-colhedora de feijão e validar o modelo de simulação do conjunto trator-colhedora de feijão desenvolvido com base em dados obtidos em testes experimentais desenvolvidos em condições de campo.

\section{MATERIAL E MÉTODOS}

Um conjunto composto por um trator e uma máquina colhedora de feijão de arrasto foi modelado e seu comportamento dinâmico simulado; posteriormente, os resultados do modelo desenvolvido foram comparados com os dados obtidos em testes experimentais, realizados em condições de campo, utilizando-se um sistema de aquisição automática de dados, validando o modelo.

O trator utilizado na simulação foi o modelo MF 620 4x2, com tração dianteira auxiliar (TDA), da Massey Ferguson. Este trator apresenta, segundo o fabricante, potência do motor na rotação nominal, de 2200 rotações min $^{-1}$ (rpm), de $77,2 \mathrm{~kW}$ (105 cv); potência máxima na TDP de $68,8 \mathrm{~kW}(93,5 \mathrm{cv})$; torque máximo no motor, na condição de $1400 \mathrm{rpm}$, de $402 \mathrm{~m} \mathrm{~N}$ (41 m kgf); massa máxima com lastro de 6000 kg; carga estática sobre o eixo dianteiro de $36 \%$; pneus dianteiros modelo R1 14,9-24 e pneus traseiros modelo R1 18,4-34.

A colhedora de feijão utilizada na simulação foi a Double Master, produzida pelas Indústrias Reunidas Colombo Ltda; trata-se de uma máquina do tipo de arrasto, que trabalha acoplada à barra de tração do trator e possui um eixo com duas rodas de sustentação. A tomada de potência do trator é utilizada para fornecer potência aos sistemas de alimentação, trilha, separação, limpeza e transporte do produto ao tanque graneleiro. Esta máquina apresenta, segundo o fabricante, potência mínima do trator para acionamento de $88 \mathrm{~kW}(120 \mathrm{cv})$; rotação nominal de trabalho de $540 \mathrm{rpm}$; largura total de 2,45 m; bitola de 2,12 m; altura de $3,25 \mathrm{~m}$; comprimento de $6,30 \mathrm{~m}$; peso total (vazia) de $3350 \mathrm{~kg}$; capacidade de produção estimada de $30 \mathrm{t} \mathrm{dia}^{-1}$ e pneus modelo R1 12,4-24.

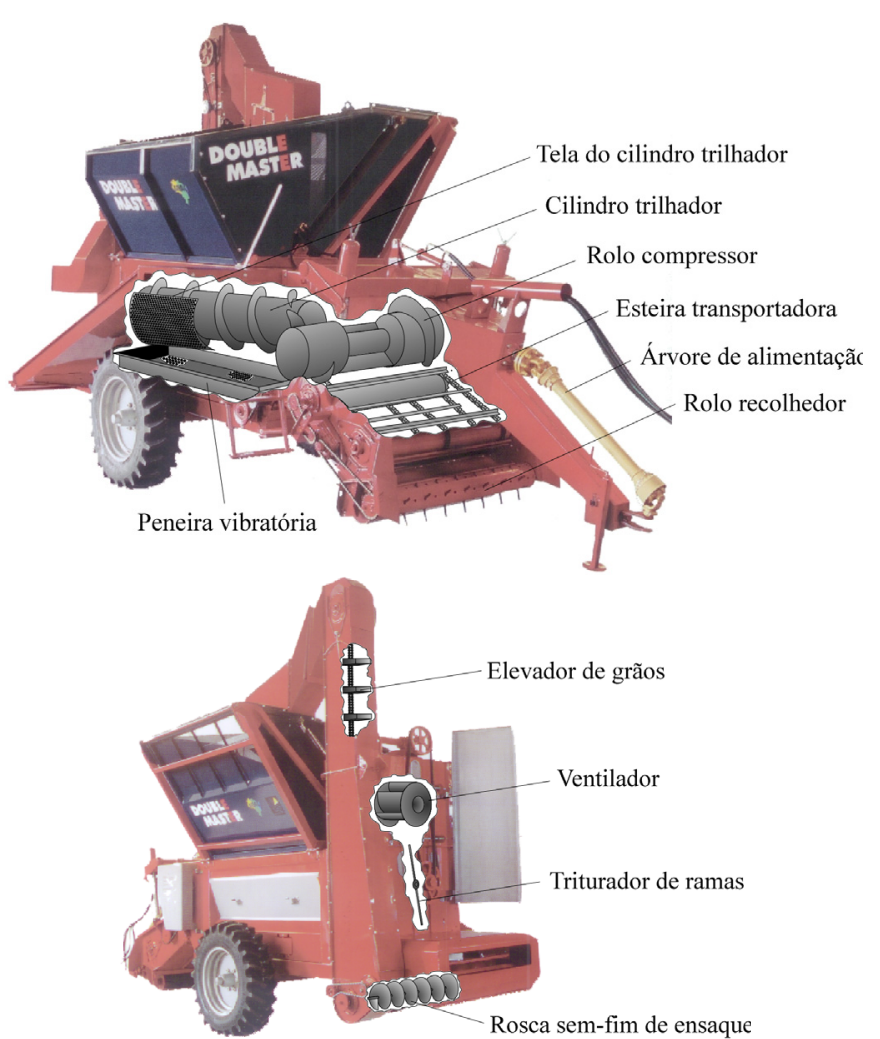

Figura 1. Principais mecanismos da colhedora de feijão Double Master

\section{Modelo matemático para simulação da dinâmica do trator}

O modelo do trator foi composto por oito corpos rígidos: a) chassi, que representa a estrutura principal do trator; b) roda traseira direita, conectada ao chassi por meio de uma junta de revolução; c) roda traseira esquerda, conectada ao chassi por meio de uma junta de revolução; d) eixo dianteiro, conectado ao chassi por meio de uma junta de revolução; e) elemento de ligação entre o eixo dianteiro e a roda dianteira direita; f) elemento de ligação entre o eixo dianteiro e a roda dianteira esquerda; g) roda dianteira direita, conectada ao elemento de ligação por meio de uma junta de revolução e $h$ ) roda dianteira esquerda, conectada ao elemento de ligação por meio de uma junta de revolução.

A força atuante em cada roda foi representada por um vetor de três componentes, um na longitudinal, outro na vertical e outro na direção lateral da roda. O componente da força longitudinal foi calculado de acordo com ASAE (1990). Para as rodas traseiras e dianteiras, a força longitudinal foi calculada empregando-se as Eqs. 1 e 2, respectivamente.

$$
F_{x t}=0,75 W\left(1-e^{-0,3 C_{n} s}\right)-W\left(\frac{1,2}{C_{n}}+0,04\right)
$$




$$
\mathrm{F}_{\mathrm{xd}}=-\mathrm{W}\left(\frac{1,2}{\mathrm{C}_{\mathrm{n}}}+0,04\right)
$$

em que:

$\mathrm{F}_{\mathrm{xt}} \quad$ - componente de força longitudinal da roda traseira, $\mathrm{N}$

$\mathrm{F}_{\mathrm{xd}}$ - componente de força longitudinal da roda traseira, $\mathrm{N}$

W - carga dinâmica atuando sobre o pneu, $\mathrm{N}$

$\mathrm{C}_{\mathrm{n}} \quad$ - coeficiente do pneu, adimensional

$\mathrm{S} \quad$ - patinagem do pneu, decimal

$\mathrm{O}$ coeficiente do pneu $\mathrm{C}_{\mathrm{n}}$ foi calculado de acordo com a equação

$$
\mathrm{C}_{\mathrm{n}}=\frac{\mathrm{CI} \mathrm{b} \mathrm{d}}{\mathrm{W}}
$$

em que:

CI - índice de cone do solo, $\mathrm{Pa}$

b - largura do pneu, $m$

d - diâmetro externo do pneu em situação indeformada, m

A simulação foi realizada considerando-se o índice de cone do solo de $830 \mathrm{kPa}$, segundo ASAE (1990) que corresponde à condição de solo firme.

O componente vertical foi representado por uma força do tipo mola-amortecedor (Eq. 4) considerando-se a força nula quando não havia contato entre a roda e o solo.

$$
\mathrm{F}_{\mathrm{z}}=\mathrm{KZ}+\mathrm{CV}_{\mathrm{z}}
$$

em que:

$\mathrm{F}_{\mathrm{z}}$ - componente de força vertical, $\mathrm{N}$

$\mathrm{K}$ - constante da mola, $\mathrm{N} \mathrm{m}^{-1}$

$\mathrm{Z}$ - coordenada vertical, $\mathrm{m}$

C - coeficiente de amortecimento, $\mathrm{N} \mathrm{s} \mathrm{m}^{-1}$

$\mathrm{V}_{\mathrm{z}} \quad$ - velocidade vertical, $\mathrm{m} \mathrm{s}^{-1}$

A constante da mola foi considerada como sendo (Lines \& Murphy, 1991):

$$
\mathrm{K}=172-1,77 \mathrm{R}+5,6 \mathrm{~A}+0,34 \mathrm{WR} \mathrm{P}
$$

em que:

$$
\begin{array}{ll}
\text { R } & \text { - largura do pneu, pol } \\
\text { A } & \text { - idade do pneu, anos } \\
\text { W } & \text { - diâmetro do pneu, pol } \\
\text { P } & \text { - pressão de trabalho do pneu, bar }
\end{array}
$$

Em todas as simulações, o valor da variável A foi considerado zero. Os valores das variáveis $\mathrm{R}$ e $\mathrm{W}$ foram considerados, para a roda dianteira, do tipo 14,9 $\mathrm{R} 24 \mathrm{e}$, para a roda traseira, do tipo 18,4 R 34 .

O coeficiente de amortecimento, considerado de acordo com Crolla et al. (1990), foi 4,3 $\mathrm{kN} \mathrm{s} \mathrm{m}^{-1}$ para os pneus dianteiros e $3,5 \mathrm{kN} \mathrm{s} \mathrm{m}^{-1}$ para os pneus traseiros.
A força lateral foi calculada por meio da equação apresentada por Crolla \& Spencer (1984):

$$
\mathrm{F}_{\mathrm{y}}=\mathrm{kW}
$$

em que:

$\mathrm{F}_{\mathrm{y}} \quad$ - componente de força lateral, $\mathrm{N}$

$\mathrm{k}^{\mathrm{y}} \quad$ - constante de proporcionalidade relativa à carga dinâmica e força lateral

$\mathrm{O}$ valor de $\mathrm{k}$ foi estimado empregando-se a seguinte equação:

$$
\mathrm{k}=-\frac{\alpha}{|\alpha|}\left[\mathrm{a}\left(1-\mathrm{e}^{-\mathrm{b}|\alpha|}\right)\right]
$$

em que:

$\alpha \quad$ - ângulo entre a direção longitudinal da roda e em direção que ela move, graus

$\mathrm{a}$ e b - coeficientes relativos à interação solo-pneu, adimensionais

A simulação foi realizada considerando-se $\mathrm{a}=0,75 \mathrm{e} \mathrm{b}=4$.

Duas equações foram desenvolvidas a partir do ensaio do motor (Quillfeldt, 2001), para estimar o torque do motor em função da velocidade angular do motor à máxima aceleração, ou

$$
\begin{gathered}
\mathrm{T}=120,29+3,5219 \omega-0,0113 \omega^{2} \\
\operatorname{para} \omega<230,4
\end{gathered}
$$

$$
\begin{gathered}
\mathrm{T}=-732765,3552+6355,4187 \omega-13,7742 \omega^{2} \\
\operatorname{para} \omega \geq 230,4
\end{gathered}
$$

em que:

$\mathrm{T}$ - torque axial do motor, $\mathrm{m} \mathrm{N}$

$\omega \quad$ - velocidade angular do motor, $\operatorname{rad~s}^{-1}$

Essas duas equações são contínuas e têm o mesmo valor de torque, em função da velocidade angular no ponto de velocidade angular do motor de 230,4 $\mathrm{rad} \mathrm{s}^{-1}$.

A relação de transmissão entre motor e rodas traseiras, segundo Massey Ferguson (2001), foi 294, 248, 193, 143, 112 , $84,75,58,45,35,27$ e 20, para a primeira até a décima-segunda marchas, respectivamente. A eficiência mecânica entre o motor e as rodas traseiras foi considerada como 0,85, segundo ASAE (1990).

\section{Modelo matemático para simulação da dinâmica da colhedora de feijão}

O modelo da colhedora de feijão desenvolvido consiste de quatro corpos rígidos: a) do chassi da colhedora, que é conectado ao chassi do trator, na posição da barra de tração usando-se uma junta esférica; b) das rodas da colhedora, que são conectadas ao chassi da colhedora por meio de juntas de 
revolução; c) da plataforma de recolhimento da colhedora, que é conectada ao chassi da colhedora por meio de uma junta de revolução e d) do tanque graneleiro da colhedora, conectado ao chassi da colhedora por meio de uma junta de revolução.

Uma força resultante especificada pela sua intensidade e sentido, foi usada para simular o atuador hidráulico responsável por descarregar o tanque graneleiro. No fundo do tanque, uma força do tipo de impacto foi aplicada, simulando-se a força que atua somente quando há contato entre o fundo do tanque e o chassi da colhedora de feijão.

As forças que atuam nas rodas da colhedora de feijão foram simuladas de forma similar às forças atuantes nas rodas dianteiras do trator.

A plataforma de recolhimento da colhedora de feijão foi suportada por uma roda rígida e uma força do tipo de impacto foi aplicada, simulando-se o contato entre a roda rígida e o solo. Duas molas localizadas entre a plataforma e o chassi da colhedora de feijão forçaram a plataforma a permanecer abaixada, em contato com o solo.

A taxa de alimentação total (palhas e grãos) colhida pela colhedora de feijão foi calculada em função do número de linhas enleiradas, espaçamento entre as fileiras, velocidade longitudinal do sistema, produtividade do feijão e da relação, em massa, de palha e grão da cultura (Eq. 10).

$$
q=\frac{P_{a} n \operatorname{ev}(1+x)}{10000}
$$

em que:

q - taxa de alimentação total da colhedora de feijão, $\mathrm{kg} \mathrm{s}^{-1}$

$\mathrm{P}_{\mathrm{a}} \quad$ - produtividade média de toda a área colhida, $\mathrm{kg} \mathrm{ha}^{-1}$

n - número de linhas enleiradas, adimensional

e $\quad$ espaçamento entre fileiras, $m$

$\mathrm{v}$ - velocidade de deslocamento, $\mathrm{m} \mathrm{s}^{-1}$

x - relação, em massa, de palha e grão, adimensional

O peso do produto no tanque graneleiro foi obtido integrando-se a taxa de alimentação do produto à máquina, em função do tempo. Uma força resultante especificada pela sua intensidade e sentido foi aplicada no centro de gravidade do tanque, representando o produto no tanque.

A potência disponível na tomada de potência foi calculada segundo a Eq. 11 proposta por ASAE (1990) para predição da demanda de potência requerida na TDP para uma colhedora de soja e grãos pequenos, com umidade de palhada de $20 \%$ de base úmida.

$$
P=7,5+7,5 q
$$

em que:

$\mathrm{P}$ - potência requerida na TDP pela colhedora, $\mathrm{kW}$

q $\quad$ - taxa de alimentação total da colhedora, $\mathrm{kg} \mathrm{s}^{-1}$

Um esquema do conjunto trator-colhedora de feijão, gerado pelo programa ADAMS, é mostrado na Fig. 2.

No modelo de simulação desenvolvido considerou-se, como parâmetro de entrada, a marcha escolhida para a realização do

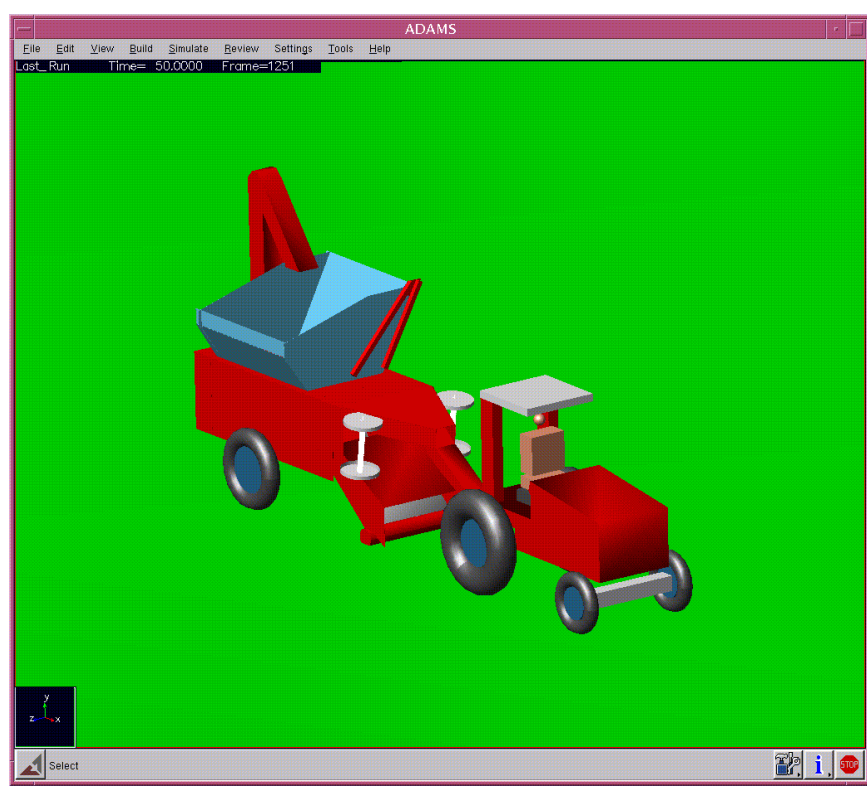

Figura 2. Esquema do conjunto trator-colhedora de feijão, desenvolvido utilizando-se o programa ADAMS

trabalho. Baseado na curva característica de torque e potência em função da rotação do motor (Quillfeldt, 2001), nas relações de transmissão do motor para a TDP e do motor para o sistema de rodado do trator MF 620 4x2 TDA (Massey Ferguson, 2001) o modelo simulou a taxa de alimentação, o torque e potência requeridos na TDP, a rotação da TDP e a velocidade de deslocamento.

O modelo do conjunto trator-colhedora de feijão foi submetido a duas diferentes condições de simulação. $\mathrm{Na}$ primeira condição simulada, a taxa de alimentação total foi calculada utilizando-se o número de linhas da leira colhida na área experimental, a produtividade da área experimental, a curva característica de torque e potência do motor, a relação de transmissão do motor, a marcha de trabalho utilizada experimentalmente e a relação, em massa, de palha e grão. Em função da variação da taxa de alimentação simulada, o modelo desenvolvido imitou o comportamento dinâmico do conjunto trator-colhedora de feijão e, na segunda condição simulada, utilizou-se a taxa de alimentação total estimada a partir de dados experimentais, como dados de entrada da taxa de alimentação simulada, interpolando os valores intermediários. Os demais parâmetros simulados foram calculados conforme a primeira condição simulada.

As comparações dos valores simulados pelo programa ADAMS e dos valores experimentais nas condições simuladas, foram realizadas eliminando-se o primeiro valor de cada série. Isto foi feito porque na simulação se considerou que o sistema partiu do repouso, enquanto nos testes experimentais a coleta de dados era iniciada já com o sistema em movimento.

\section{RESULTADOS E DISCUSSÃO}

As comparações dos valores simulados pelo programa ADAMS, sob duas diferentes condições de simulação, e dos valores experimentais da variação da velocidade de deslocamento, da taxa de alimentação estimada, da rotação da TDP, do torque e da potência requeridos na TDP, em função do tempo, são apresentadas a seguir. 
A primeira condição simulada é apresentada nas Figura 3 (A, B, C, D e E); na Figura 3A, apresenta-se a comparação entre as velocidades de deslocamento simulada e experimental e se
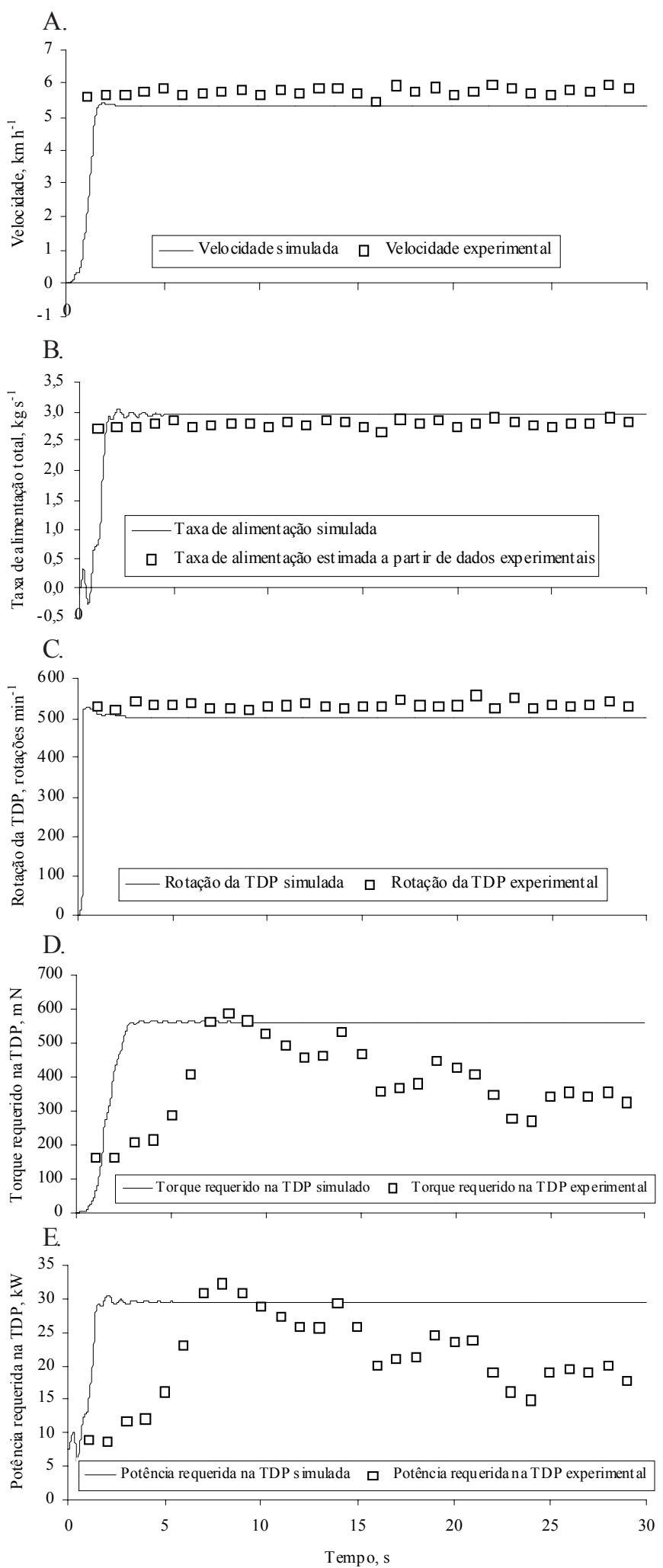

Figura 3. Comparação entre velocidade de deslocamento (A), taxa de alimentação total $(B)$, rotação da TDP $(C)$, torque requerido na TDP (D) e potência requerida na TDP (E) simuladas pelo programa ADAMS e obtidas experimentalmente observa que o modelo subestimou os dados experimentais com erro relativo médio de $8,35 \%$; tal comportamento pode ser atribuído à possibilidade da taxa de alimentação total simulada ter sido superior à experimental. Este fato ocorreu devido a taxa simulada ter sido calculada em função da produtividade média de toda a área colhida, enquanto a taxa experimental foi medida sob condição de menor produtividade, resultando num acréscimo maior da carga resistiva nos mecanismos da colhedora e, conseqüentemente, em maior decréscimo de rotação da TDP simulada.

A Figura 3B apresenta a comparação entre as taxas de alimentação total simulada e estimada a partir de dados experimentais. Observa-se que a taxa de alimentação simulada foi superior àquela estimada a partir dos dados de número de linhas, espaçamento, produtividade e velocidade obtidos, experimentalmente, com erro relativo médio de 4,99\%. Tal erro pode ser atribuído ao fato da taxa de alimentação total simulada ter sido calculada em função da produtividade média de toda a área colhida, enquanto a estimada a partir de dados experimentais considerou apenas determinada parcela experimental em que ocorreu uma produtividade inferior $\left(1,84 \mathrm{t} \mathrm{ha}^{-1}\right)$ à média de toda a área colhida $\left(2,18 \mathrm{t} \mathrm{ha}^{-1}\right)$. A taxa de alimentação total, estimada a partir de dados experimentais, foi determinada em função da variação da velocidade de deslocamento, considerando-se a produtividade da leira colhida constante, ao longo do ensaio, uma vez que a máquina colhedora não dispunha de instrumentos para medir a taxa de alimentação de feijão, em tempo real.

A comparação entre as rotações da TDP simulada e experimental é apresentada na Figura 3C, na qual se observa que o modelo subestimou os dados experimentais com erro médio de $6,02 \%$, cujo comportamento pode ser atribuído à possibilidade da taxa de alimentação total simulada ter sido superior à experimental, o que resultou em maior acréscimo de carga resistiva nos mecanismos da colhedora e, conseqüentemente, em maior decréscimo de rotação da TDP simulada, em relação aos valores experimentais observados.

$\mathrm{Na}$ Figura 3D tem-se a comparação entre os torques requeridos na TDP simulado e experimental. Observa-se que o modelo apresentou erro relativo médio de $30,61 \%$, podendo-se observar que se aproximou do valor máximo de torque experimental e gerou valores de torque praticamente constantes, após $5 \mathrm{~s}$, devido ao fato da taxa de alimentação simulada ter tido o mesmo comportamento ao longo do ensaio. Constata-se grande variação do torque experimental. Este comportamento pode ser atribuído ao fato da leira ter apresentado desuniformidade ao longo do ensaio, diminuindo seu volume de palha e grão, no final da leira colhida. Não foi observada relação entre os valores de torque e a taxa de alimentação estimada a partir de dados experimentais, pelo fato do torque observado ser resultante da resistência imposta pela real condição da leira colhida. A taxa de alimentação total estimada a partir de dados experimentais, contudo, foi calculada desconsiderando-se a real condição da leira colhida. O modelo gerou valores de torque seguindo o comportamento de variação da taxa de alimentação total simulada.

A Figura 3E mostra a comparação entre as potências requeridas na TDP simulada e experimental. Encontrou-se um 
erro relativo médio de $27,80 \%$ e se observou que as potências simulada e experimental acompanharam as variações dos torques simulado e experimental, respectivamente. Este comportamento pode ser atribuído ao fato da potência ter sido calculada em função da variação do torque e rotação da TDP.

O elevado erro relativo médio observado, também pode ser atribuído ao fato de ter sido utilizada, no programa ADAMS, uma equação para predição de potência requerida na TDP para uma colhedora de soja e grãos pequenos, para material de palhada com umidade de $20 \%$ (base úmida). A cultura usada nos ensaios foi o feijão com umidade média inferior a $20 \%$. Esta equação foi empregada pelo fato de ser a única disponível para predição da potência, para cultura semelhante à do feijão, uma vez que este também é uma leguminosa como a soja. Outro fato que pode ter contribuído para o elevado erro relativo médio foi de ter sido utilizada, na simulação, a taxa de alimentação total estimada a partir da produtividade média de toda a área colhida, ao invés da taxa de alimentação total real.

Pode-se observar que todos os gráficos comparativos apresentam os valores simulados partindo do valor zero, o que se pode atribuir ao fato do trator, que tracionava a colhedora de feijão, ter iniciado a simulação com o motor em estado de repouso. Contudo, o ensaio experimental apresenta valores altos a partir do início, pelo fato do conjunto trator-colhedora ter sido colocado em movimento antes de se iniciar a aquisição de dados.

A segunda condição simulada é apresentada nas Figura 4 (A, B, C, D e E); na Figura 4A tem-se a comparação entre as velocidades de deslocamento simulada e experimental; observa-se que o modelo subestimou os dados experimentais, com erro relativo médio de $8,18 \%$, comportamento que pode ser atribuído ao fato da taxa de alimentação total simulada ter sido considerada igual à estimada a partir de dados experimentais. Esta taxa estimada foi determinada em função da variação da velocidade de deslocamento, considerando-se a produtividade da leira colhida constante ao longo do ensaio, uma vez que a máquina colhedora não dispunha de instrumentos para medir a taxa de alimentação de feijão, em tempo real. Na verdade, a taxa de alimentação total experimental deve ter sido diferente da estimada a partir de dados experimentais e, possivelmente, foi inferior, o que pode ter causado menor resistência nos mecanismos da colhedora, aumentando a rotação do motor e, conseqüentemente, a velocidade de deslocamento experimental, em relação aos valores simulados observados.

A Figura 4B mostra a comparação entre as taxas de alimentação total simulada e estimada a partir de dados experimentais. Os dados simulados e os calculados de dados experimentais são iguais, em razão dos valores simulados terem sido considerados como se fossem os calculados sob esta condição de modelagem. Os valores intermediários foram interpolados pelo modelo desenvolvido.

A comparação entre as rotações da TDP simulada e experimental é apresentada na Figura 4C. Vê-se que o modelo subestimou os dados experimentais, com erro médio de 5,85\%, cujo comportamento pode ser atribuído ao fato da rotação da TDP simulada ser função da taxa de alimentação total simulada, que foi diferente e, possivelmente, maior que a experimental, o

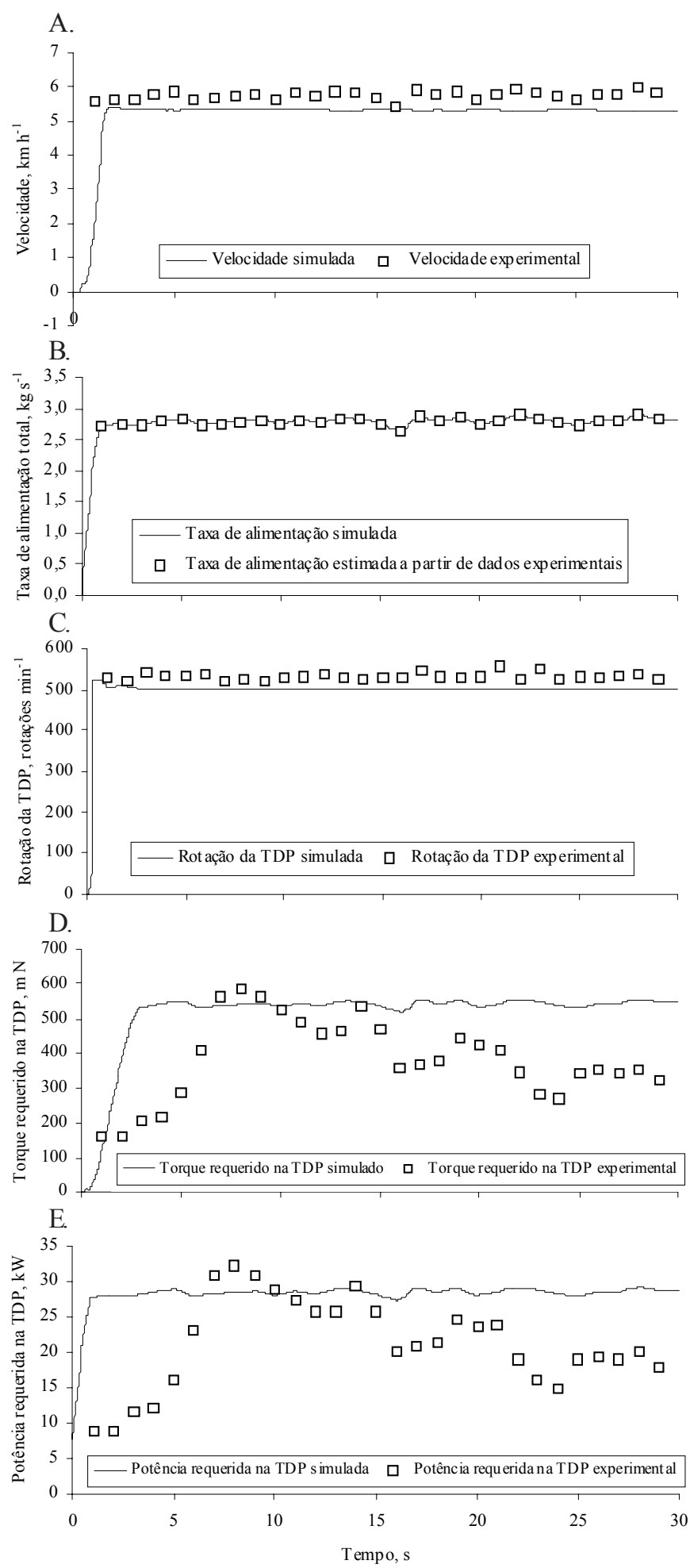

Figura 4. Comparação entre velocidade (A), taxa de alimentação (B), rotação da TDP $(C)$, torque requerido na TDP (D) e potência requerida na TDP (E) simuladas pelo programa ADAMS obtidas experimentalmente

que pode ter causado maior resistência nos mecanismos da colhedora, reduzindo a rotação da TDP simulada em relação aos valores experimentais observados.

A Figura 4D apresenta a comparação entre os torques requeridos na TDP simulado e experimental, constatando-se que o modelo apresentou erro relativo médio de $28,69 \%$. 
Verifica-se que o modelo apresentou os valores variando dentro dos valores de torque experimental. A variação do torque experimental pode ser atribuída à variação da taxa de alimentação total experimental, que era desuniforme. O modelo gerou valores de torque, seguindo o comportamento de variação da taxa de alimentação total simulada.

A Figura 4E apresenta a comparação entre as potências requeridas na TDP simulada e experimental. Encontro-se um erro relativo médio de $26,23 \%$. Observa-se que as potências simulada e experimental acompanharam as variações de torques simulado e experimental, respectivamente, atribuindo-se ao fato da potência ter sido calculada em função da variação do torque e rotação da TDP.

\section{CONCLUSÕES}

1. Um modelo matemático para simular o comportamento dinâmico do conjunto trator-colhedora de feijão, foi desenvolvido e utilizado sob três diferentes condições. $\mathrm{O}$ modelo de simulação foi validado comparando-se os dados obtidos sob testes experimentais desenvolvidos em condições de campo, com os valores obtidos na simulação.

2. Para melhor condição de simulação observou-se erro relativo médio de $8,18,5,85,28,69$ e $26,23 \%$ para a velocidade de deslocamento, rotação da TDP, torque requerido na TDP e potência requerida na TDP, respectivamente. Em outra condição de simulação, o modelo simulou melhor a taxa de alimentação total, observando-se erro relativo médio de 4,99\%.

3. Com base nos resultados, conclui-se que o modelo de simulação desenvolvido ainda requer alterações e aprimoramento, com o objetivo de se reduzir os erros observados.

\section{AGRADECIMENTOS}

Ao Conselho Nacional de Desenvolvimento Científico e Tecnológico (CNPq), pela bolsa concedida. À Fundação de Amparo à Pesquisa do Estado de Minas Gerais (FAPEMIG) e ao Programa Redes Cooperativas de Pesquisa (RECOPE/FINEP), pelo auxílio à pesquisa e suporte financeiro. À EMBRAPA Milho e Sorgo e à EPAMIG de Sete Lagoas, pelo suporte na realização de testes experimentais. Às Indústrias Reunidas Colombo, pelo suporte financeiro.

\section{LITERATURA CITADA}

ASAE - American Society of Agricultural Engineering. D497Agricultural machinery management data. In: Standards 1990. St. Joseph: ASAE, p.285-291. 1990.

Brasil. Ministério da Agricultura e do Abastecimento. Companhia Nacional de Abastecimento. Política Agrícola - SAFRA. Disponível em: < http://www.conab.gov.br/politica_agricola/ safra/avalia.html>. Acesso em: 15 janeiro 2002.

Conto, A.J.; Vieira, E.H.N.; Oliveira, E.T.; Castro, T.A.P. Aspectos técnicos e econômicos da colheita mecânica e manual do feijão (Phaseolus vulgaris L.). Goiânia, GO: EMBRAPACNPAF, 1980. 14p.

Crolla, D.A.; Horton, D.N.L.; Stayner, R.M.; Effect of tire modeling on tractor ride vibration predictions. Journal of Agricultural Engineering Research, London, v.47, p.55-77, 1990.

Crolla, D.A.; Spencer, H.B. Tractor handling during control loss on slopping ground. Vehicle system dynamics. Lisse, Netherland, v.13.p.1-17.1984.

Kim, K.U.; Hoag, D.L.; Hunt, D.R. Ride simulation of passive, active, and semi-active seat suspensions for off-road vehicles. Transactions of the ASAE. St. Joseph, v.28, n.1, p.56-64. 1985.

Lines, J.A.; Murphy, K. The stiffness of agricultural tractor tires. Journal of Terramechanics, Great Britain: Tergaman Press, v.28, n.1, p.49-64. 1991.

Massey Ferguson. MF620: MF620/4 Especificações técnicas. Disponível em: <http://www.massey.com.br/portugues/ espProduto.asp?idproduto $=551 \&$ serie $=6>$. Acesso em: 28 novembro 2001.

McGrath, J.F. Numerical methods in ADAMS: Advanced ADAMS/Solver course. Ann Arbor, MI, 1994. 435p.

Queiroz, D.M. Steering performance simulation of rubbertracked tractor. West Lafayette: Purdue University, 1995. 233p. PhD Thesis

Quillfeldt, W. Curva característica do motor P4000T. [mensagem pessoal]. Mensagem recebida por<garcia@ufv.br> em 29 novembro 2001 